\title{
Erratum to: Serum concentrations of PCBs and OCPs among prepubertal Korean children
}

\author{
Su Hyun Park ${ }^{1}$ - Young Sun Hong ${ }^{2} \cdot$ Eun-Hee Ha ${ }^{1} \cdot$ Hyesook Park ${ }^{1}$
}

Published online: 17 February 2016

(C) Springer-Verlag Berlin Heidelberg 2016

Erratum to: Environ Sci Pollut Res (2016) 23:3536-3547

DOI 10.1007/s11356-015-5578-0

The original publication of this article contains a mistake. Acknowledgments section should read: This research was supported by a grant (13162MFDS891) from the Ministry of Food and Drug Safety in 2013.

The online version of the original article can be found at http://dx.doi.org/ 10.1007/s11356-015-5578-0.

Hyesook Park

hpark@ewha.ac.kr

Su Hyun Park

shp323@ewha.ac.kr

Young Sun Hong

imhys@ewha.ac.kr

Eun-Hee Ha

eunheeha@ewha.ac.kr

1 Department of Preventive Medicine, School of Medicine, Ewha Womans University, 1071, Anyangcheon-ro, Yangcheon-ku, Seoul 158-710, Republic of Korea

2 Department of Internal Medicine, School of Medicine, Ewha Womans University, Seoul, Korea 